\title{
Coronary Artery Fistula Unmasking the Absence of Left Pulmonary Artery in an Adult
}

\author{
Holger H. Sigusch ${ }^{1}$ Andreas Hansch ${ }^{2}$ Torsten Doenst ${ }^{3}$ \\ ${ }^{1}$ Division of Cardiology, Department of Internal Medicine, Heinrich- \\ Braun-Klinikum, Zwickau, Germany \\ 2 Department of Radiology, Interventional Radiology and \\ Neuroradiology, Heinrich-Braun-Klinikum, Zwickau, Germany \\ 3 Department of Cardiothoracic Surgery, Jena University Hospital, \\ Friedrich-Schiller-University of Jena, Jena, Germany \\ Thorac Cardiovasc Surg Rep 2020;9:e9-e10.
}

\begin{abstract}
Address for correspondence Holger $\mathrm{H}$. Sigusch, MD, Division of Cardiology, Department of Internal Medicine, Heinrich-BraunKlinikum-Zwickau, Karl-Keil-Strasse 35, 08060 Zwickau, Germany (e-mail: holger.sigusch@hbk-zwickau.de).
\end{abstract}

Abstract
Keywords
- congenital defect
- myocardial infarction
- pulmonary arteries

Coronary artery fistulae are an incidental finding in patients undergoing coronary angiography or computed tomography (CT) coronary angiography. A 60-year-old man with known coronary artery disease presented with dyspnea. Coronary angiography revealed a large fistula arising from the circumflex artery (CX) without a clear intrathoracic target vessel or chamber in the heart. CT angiography revealed the agenesis of the left pulmonary artery. The fistula arising from the $C X$ ensured left lung tissue supply. Unilateral absence of a pulmonary artery is an extremely rare condition. In this case, the identification of a fistula from the heart triggered the correct diagnosis.

\section{Introduction}

Coronary artery fistulae are usually abnormal connections between a coronary artery and a cardiac chamber or major intrathoracic vessels with substantially lower pressure. Most coronary fistulae are congenital, but they can also be acquired. In the largest series of cases undergoing coronary angiography, relevant coronary fistulae were found in 62 out of 126,595 cases $(0.05 \%) .{ }^{1}$ More recent studies by computed tomography (CT) coronary angiography, screening $15,548,8,864$, and 2,573 patients found higher incidences of $0.19,0.15$, and $0.15 \%$, respectively. $^{2-4}$ Most patients are asymptomatic. The most common findings in symptomatic patients are heart failure resulting from left to right shunting, ischemia due to coronary steal, arrhythmia, rupture, thrombosis, and infective endocarditis. ${ }^{5}$

\section{Case Description}

A 60-year-old man suffered a non-ST-segment elevation myocardial infarction at the age of 49 years (year 2009) and underwent interventional treatment of the left anterior descending artery (LAD, bare metal stent implantation) and mechanical recanalization (balloon only) of the ramus intermedius. At this time, intervention via the femoral route was challenging because of difficulty to selectively intubate the ostium of the left coronary artery and challenging wire passage into the LAD. A large coronary fistula ( - Fig. 1, panel A) arising from the circumflex artery (CX) had already been described, but at that time, no further action had been taken. Now, more than 10 years later, the patient returned with exertional dyspnea and again underwent coronary angiography. LAD and ramus intermedius showed intermediate lesions without any further progress of his coronary artery disease. Since he was symptomatic, but his lesions did not appear flow limiting, interventional closure of the fistula was considered, but a CT angiography of the chest was performed to further evaluate the fistula and its target vessel or chamber. His chest CT showed a smaller left and larger right hemithorax together with fibrotic and emphysematous changes of the left lung ( - Fig. 1, panel B). The underlying cause was an agenesis of the left pulmonary artery ( - Fig. 1, panel B). Collaterals from the heart (fistula coming from the CX), the left and right subclavian arteries, as well as the superior phrenic artery received

October 28, 2019

accepted after revision

December 13, 2019
DOI https://doi.org/

10.1055/s-0040-1702212. ISSN 2194-7635.
๑) 2020 Georg Thieme Verlag KG
Stuttgart · New York

License terms

(®) (1) $\circledast$ 

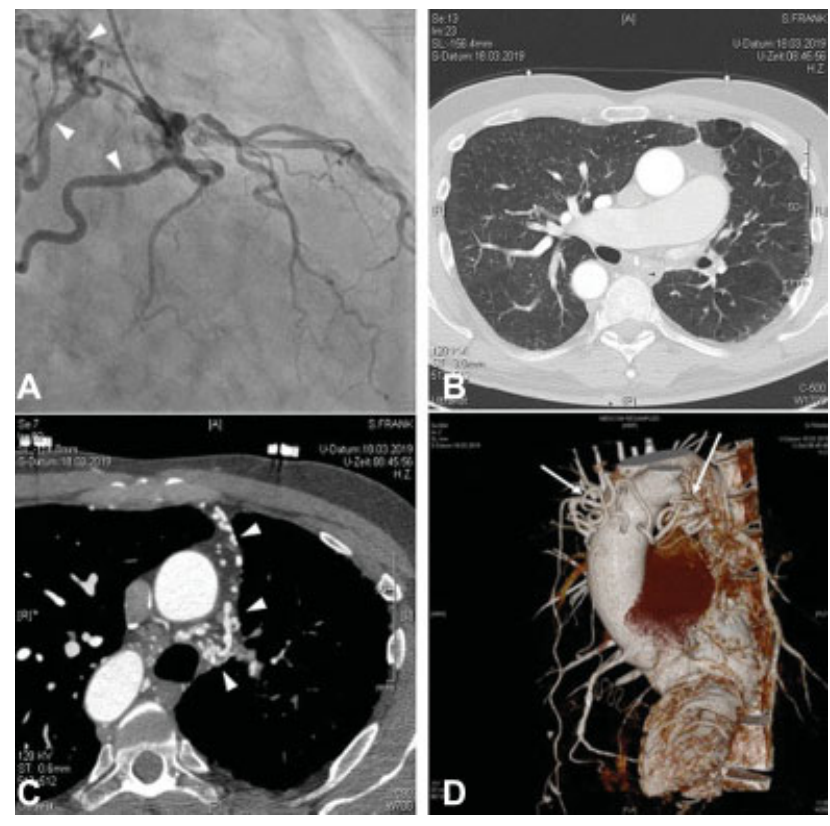

Fig. 1 Coronary angiography in right anterior oblique 30-degree projection showing intermediate lesions of the proximal left anterior descending artery and the ramus intermedius as well as a large "fistula" (arrow heads) taking off from the circumflex artery (panel A); CT of the chest indicating a smaller left hemithorax and the absence of the left pulmonary artery (panel B); CT angiography showing the right descending aorta and multiple collaterals (arrow heads) close to the ascending aorta (panel C); and threedimensional reconstruction of the intrathoracic structures with large collateral vessels arising from both subclavian arteries (arrows, panel D). CT, computed tomography.

(-Fig. 1, panels C and D) ensured left lung tissue supply. In addition, it revealed a right descending thoracic aorta. Detailed history revealed dyspnea since childhood which led to discharge from military service later in life due to hemoptysis induced by maximum strength exercise. The decision for conservative treatment was made.

\section{Discussion}

Unilateral absence of a pulmonary artery (UAPA) is an extremely rare condition affecting $\sim 1$ in 250,000 persons in the general population and is mostly associated with other congenital defects and is mainly diagnosed in childhood. ${ }^{6-9}$ In the studies searching for coronary anomalies in $\sim 175,000$ patients, not a single case of UAPA had been described, underlining the uniqueness of this case. ${ }^{1-4}$
Here, a large fistula arising from the CX triggered CT coronary angiography and thereby the diagnosis. It showed that the "fistula" had no clear target vessel or target cardiac chamber but corresponded to collaterals ensuring tissue integrity of the left lung. Since these collaterals supplied the tissue of the left lung, an interventional closure was not indicated. In addition, his coronary lesions were not flow limiting. Thus, the patient's dyspnea was most likely caused by the congenital defect (UAPA) and associated consequences rather than by a steal phenomenon at the level of the heart. Fistulae arising from the coronary tree which do not have a clear target vessel or target cardiac chamber should therefore be evaluated by CT coronary angiography before considering interventional or surgical closure.

Conflict of Interest

None.

\section{References}

1 Yamanaka O, Hobbs RE. Coronary artery anomalies in 126,595 patients undergoing coronary arteriography. Cathet Cardiovasc Diagn 1990;21(01):28-40

2 Zhou K, Kong L, Wang Y, et al. Coronary artery fistula in adults: evaluation with dual-source CT coronary angiography. Br J Radiol 2015;88(1049):20140754

3 Namgung J, Kim JA. The prevalence of coronary anomalies in a single center of Korea: origination, course, and termination anomalies of aberrant coronary arteries detected by ECG-gated cardiac MDCT. BMC Cardiovasc Disord 2014;14:48

4 Graidis C, Dimitriadis D, Karasavvidis V, et al. Prevalence and characteristics of coronary artery anomalies in an adult population undergoing multidetector-row computed tomography for the evaluation of coronary artery disease. BMC Cardiovasc Disord 2015; $15: 112$

5 Jama A, Barsoum M, Bjarnason H, Holmes DR Jr, Rihal CS. Percutaneous closure of congenital coronary artery fistulae: results and angiographic follow-up. JACC Cardiovasc Interv 2011;4(07):814-821

6 Weldetsadik AY, Asfaw YM, Tekleab AM. Isolated absence of right pulmonary artery in a 4-year old child: a case report. Int Med Case Rep J 2018;11:297-301

7 Steiropoulos P, Archontogeorgis K, Tzouvelekis A, Ntolios P, Chatzistefanou A, Bouros D. Unilateral pulmonary artery agenesis: a case series. Hippokratia 2013;17(01):73-76

8 De Dominicis F, Leborgne L, Raymond A, Berna P. Right pulmonary artery agenesis and coronary-to-bronchial artery aneurysm. Interact Cardiovasc Thorac Surg 2011;12(03):507-509

9 Koga H, Hidaka T, Miyako K, Suga N, Takahashi N. Age-related clinical characteristics of isolated congenital unilateral absence of a pulmonary artery. Pediatr Cardiol 2010;31(08):1186-1190 